\title{
A model of $\mathrm{N}$-terminal Cyclin $\mathrm{T} 1$ based on FRET experiments
}

\author{
SERGIO PANTANO $+\| \#$, ALESSANDRO MARCELLO $¥ \#$, ARIANNA SABÒ§, ALDO FERRARIף, VITTORIO PELLEGRINIף, \\ FABIO BELTRAMף §, MAURO GIACCA $\uparrow \S *$ and PAOLO CARLONI $† * *$ \\ $\dagger$ International School for Advanced Studies (ISAS) and INFM-DEMOCRITOS Modeling Center for Research in Atomistic Simulation, Via Beirut 2-4, \\ 34014 Trieste, Italy \\ †International Center for Genetic Engineering and Biotechnology (ICGEB), Padriciano 99, 34012 Trieste, Italy \\ ๆ National Enterprise for nanoScience and nanoTechnology-Istituto Nazionale di Fisica della Materia (NEST-INFM), Via della Faggiola 17, 56126 \\ Pisa, Italy \\ $\S$ Scuola Normale Superiore, Piazza dei Cavalieri 7, 56100 Pisa, Italy \\ \|Venetian Institute of Molecular Medicine (VIMM), Via Orus 2, 35129, Padua, Italy
}

\begin{abstract}
Human Cyclin T1 is the cyclin partner of kinase CDK9 in the positive transcription elongation factor b $(\mathrm{P}-\mathrm{TEFb}) . \mathrm{P}-\mathrm{TEFb}$ is recruited by Tat, the transactivator of the human immunodeficiency virus type 1 (HIV-1), to the viral promoter by direct interactions between Tat, Cyclin T1 and the cis-acting transactivation-responsive region (TAR) present at the $5^{\prime}$-end of each viral mRNA. At present, no structural data for Cyclin T1 are available. Here, we build a structural model of an N-terminus portion of Cyclin T1 (aa 27-263) based on the X-ray structure of Cyclin H. The model is compared with site directed mutagenesis data from the literature and validated by fluorescence resonance energy transfer (FRET) using Tat as a probe in living cells. This model provides a first step towards the structural characterization of the CDK9-CycT1-Tat-TAR complex, which is crucial for HIV-1 replication and may constitute a promising target for pharmaceutical intervention.
\end{abstract}

Keywords: Cyclin T1; HIV-1; Tat; FRET; Comparative modelling; P-TEFb

\section{Introduction}

The cyclin family of proteins plays a fundamental role in the cell cycle functioning as regulatory subunits of the socalled cyclin dependent kinases (CDK), which act as catalytic subunits [1]. Besides regulation of cell cycle by phosphorylation of a large variety of substrates, Cyclin/CDK complexes are also involved in the control of different cellular mechanisms such as differentiation, apoptosis and, for some members of the family, such as Cyclin $\mathrm{H}(\mathrm{CycH})$, Cyclin $\mathrm{C}(\mathrm{CycC})$ and Cyclin $\mathrm{T} 1$ (CycT1), transcription initiation and/or elongation [2].

The common structural feature of the family is the cyclin box motif. This is a characteristic two-repeats folding motif of $\sim 100$ amino acids long [3,4] that constitute a versatile scaffold for mediating proteinprotein interactions. Each repeat is composed of five helices, connected by a linker peptide in extended conformation. The helices within each repeat are spatially disposed with the very hydrophobic helix H3 surrounded by the other four. Remarkably, this motif bears a high degree of structural conservation even at very low primary sequence identity. The relative orientation of the repeats can vary as found for the transcription factor II B (TFIIB) that shows a different arrangement. Cyclin boxes are usually inserted into a protein frame, with additional helical elements found at the $\mathrm{N}$ and $\mathrm{C}$ termini of the cyclin box. These segments are not conserved among the cyclin family and they likely provide binding specificity for protein-protein interactions.

Cyclin T1 is the cyclin associated to CDK9 in the positive transcription-elongation factor $\mathrm{b}(\mathrm{P}-\mathrm{TEFb})$, a general elongation co-factor for RNA polymerase II (RNAPII) directed transcription [34]. P-TEFb is able to phosphorylate the carboxy-terminal domain (CTD) of RNAPII, a molecular event associated with increased transcriptional processivity. Initially, CycT1 has been discovered as a cofactor of the human immunodeficiency virus type 1 (HIV-1) Tat transactivator [5]. A tripartite complex is formed between Tat, CycT1 and the cis-acting

\footnotetext{
\#These authors contributed equally to the work.

*Corresponding authors. Mauro Giacca, International Center for Genetic Engineering and Biotechnology (ICGEB), Padriciano 99, 34012 Trieste, Italy. Tel.: + 39-040-3757324. Fax: +39-040-226555. Email: giacca@icgeb.org

Paolo Carloni, International School for Advanced Studies, Via Beirut 4, 34014 Trieste, Italy. Tel.: + 39-040-3787-407. Fax: + 39-040-3787-528. Email: carloni@sissa.it
} 
transactivation-responsive region (TAR) present at the $5^{\prime}$ end of each viral mRNA. The formation of the P-TEFbTAR-Tat complex is an essential step towards the assembly of the processive RNAPII machinery at the LTR promoter [6-9]. CycT1 can be grossly sub-divided into two major domains (figure $1(\mathrm{~A})$ ). The $\mathrm{N}$ terminus of the protein (aa 1-300) shares partial homology with other members of the Cyclin T family (cyclin box) [5]. It contains the Tat recognition motif (TRM) that includes a critical cysteine residue at position 261 present in human CycT1, but absent in its rodent homologue $[6,8]$. The $\mathrm{N}$ terminus domain of CycT1 is also important for the protein-protein associations of the protein with CDK9, NF- $\kappa$ B, CIITA and c-Myc [10-12]. The C terminus of CycT1 is less well characterized and contains a putative coiled-coil region (aa 379-430; coil); a histidine-rich domain (aa 506-530; His) and a carboxy-terminus PEST sequence (aa 700-726). It has been recently shown that the $\mathrm{C}$ terminus of CycT1 is involved in the association with the CTD domain of RNAPII [13,14] and the promyelocytic leukaemia protein, PML [15]. At present, no three-dimensional structure of CycT1 is available. Here, we use bioinformatics tools to model the $\mathrm{N}$ terminus (aa 27-263) of CycT1. We build the structural model based on the X-ray structure of $\mathrm{CycH}$, another member of the cyclin family involved in transcription, whose X-ray structure is available. The model fits with all mutagenesis data present in the literature. Furthermore, we take advantage of the complex interactions between the HIV-1 Tat protein and CycT1 in vivo to validate the prediction of close proximity between the $\mathrm{N}$ and $\mathrm{C}$ termini parts of the Cyclin box. Specifically, we measure the fluorescence resonance energy transfer (FRET) between fluorescent proteins genetically fused to the $\mathrm{N}$ and $\mathrm{C}$ termini of Cyclin $\mathrm{T} 1$ and to the $\mathrm{C}$ termini of Tat in living cells.

A

Cyclin T1

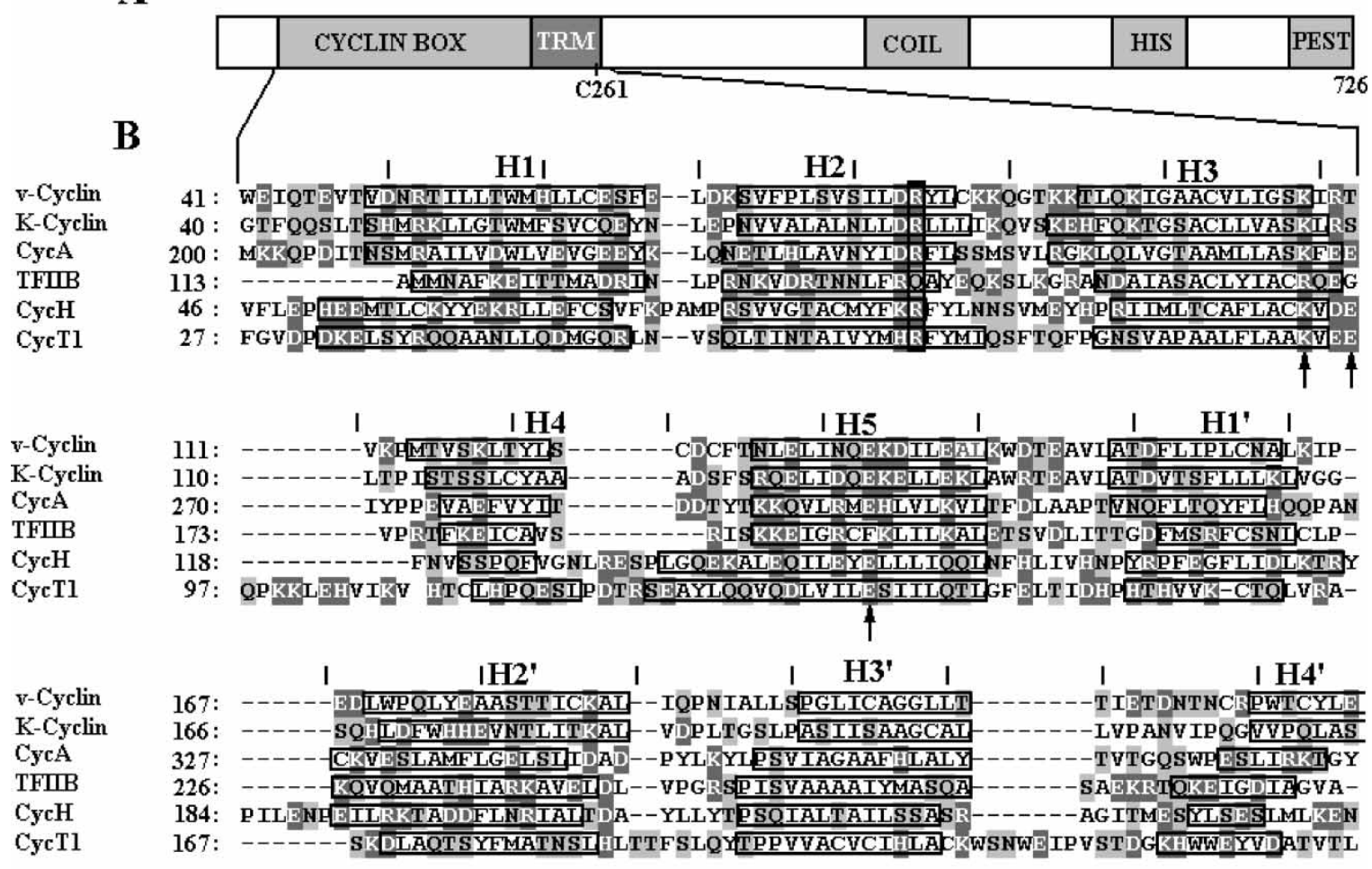

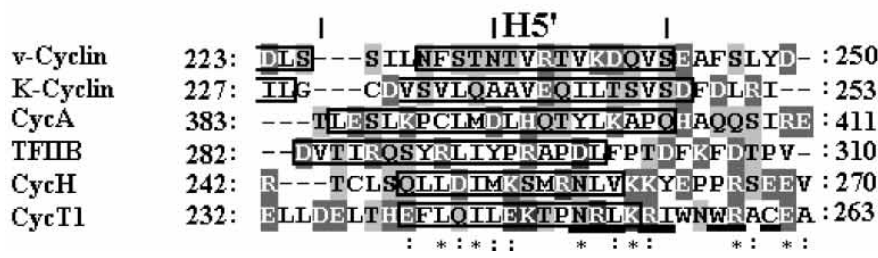

Figure 1. Alignment of Cyclin-boxes. (A) Domains representation of CycT1. (B) Sequence alignment of the cyclin box of CycT1 with the structural templates. The chemical character of the residues is indicated by background colours. Black characters in white background correspond to hydrophobic aminoacids, light grey are polar and dark grey with white characters are charged residues. Black rectangles indicate helical elements as found in crystallographic structures. The conserved arginines that fix the relative orientation of the repeats in all but TFIIB proteins is indicated with a vertical rectangle. Arrows indicate residues whose charge inversion by mutagenesis inhibits CycT1:CDK9 binding. Residues belonging to the Tat recognition motif are underlined. Homology between C-terminal motifs of $\mathrm{CycH}$ and $\mathrm{CycT} 1$ are indicated with stars for identities and colons for conservative mutations. 


\section{Methods}

\subsection{Bioinformatics}

The protocol used for the construction of the structural model of CycT1 (Swiss Prot entry O60563, [5]) was the following:

2.1.1 Identification of appropriate templates. We considered a set of five non redundant cyclin proteins whose structure was determined by X-ray crystallography: (i) a viral cyclin (v-cyclin) from herpesvirus saimiri, homolog to type $\mathrm{D}$ cyclins, able to specifically activate human CDK6 (PDB code:1BU2) [16]; (ii) a viral D-type cyclin encoded by the Kaposi's sarcoma-associated herpesvirus (K-cyclin, PDB code:1G3N) [17]; (iii) the human Cyclin A (CycA, PDB code:1FIN) [18]; (iv) the general transcription factor II B (TFIIB) [19] (PDB code: $1 \mathrm{VOL})$ and (v) the human transcriptional cyclin $\mathrm{H}$ (PDB code:1JKW) [20].

2.1.2 Sequence alignment. Primary sequences of the above-mentioned cyclins were initially aligned using T-COFFEE (http://www.ch.embnet.org/software/TCoffee. html), which was shown to be more efficient in case of low sequence identity [21]. The alignments were then manually refined so as to take into account the secondary structure data.

2.1.3 Selection of the structural template. Among the above quoted structural templates, $\mathrm{CycH}$ is the only one human protein involved in transcription activation as CycT1. In addition, $\mathrm{CycH}$ was also reported to interact with Tat [22]. Indeed, the C-terminal segment of $\mathrm{CycH}$ shares high homology with its corresponding part in CycT1 (figure 1) that contains the TRM. For these reasons, $\mathrm{CycH}$ was chosen as structural template to coordinate mapping to our target CycT1. The X-ray structure of $\mathrm{CycH}$ was determined at a resolution of $2.6 \mathrm{~nm}$ with 269 residues traced corresponding to two cyclin box repeats (about 100 amino acids each). Before and after the cyclin box two $\mathrm{N}$ and $\mathrm{C}$ terminal helical motifs, $H_{\mathrm{N}}$ and $H_{\mathrm{C}}$ of 21 and 15 residues long, respectively complete the structure of $\mathrm{CycH}$.

2.1.4 Construction of the structural models. Although CycT1 is 726 amino acids long, deletion mutant experiments shown only the $\mathrm{N}$ terminus 1-263 [23] amino acids are needed to bind CDK9 and Tat. Our model is based on the structure of $\mathrm{CycH}$ from residues 46 to 270 . This fragment was mapped to amino acid 27 to 263 of CycT1. Therefore, it covers the necessary protein length relevant for the study of the CycT1 with respect to its functional association with CDK9 and Tat. The construction and refinement of the backbone and side chains of the target protein were performed with the program Modeller V6.2 [24,25].
2.1.5 Selection of the best model. The best structural model was based on Modeller's restraints violation. The model selected (figure 2) exhibited a good Ramachandran map, with $93.9 \%$ of the residues in most favoured regions, $5.2 \%$ in allowed regions and only $0.8 \%$ (Ser188 and Thr230) in unfavourable regions.

\subsection{Experimental methods}

2.2.1 Plasmids and cells. The expression plasmids pcDNA3-Tat-BFP and pcDNA3-EGFP-CycT1, containing EGFP fused at their $3^{\prime}$ - and $5^{\prime}$-ends, respectively, were characterized previously [26]. The plasmid pCycT300EGFP, carrying EGFP at the $3^{\prime}$ end, was obtained by PCR amplification of the $\mathrm{N}$ terminus of CycT1 with primers: $\mathrm{FW}$ $5^{\prime}$-gc gaa ttc atg gag gga gag agg aag aac- $3^{\prime}, \mathrm{RV} 5^{\prime}$-gc gga tcc ag gta att tgg acg tta cca- $3^{\prime}$ and cloning as an EcoRI/BamHI fragment into pEGFP-N1 (Clontech). The plasmid pBFPCycT300-EGFP, carrying BFP at the $5^{\prime}$-end and GFP at the $3^{\prime}$-end, was obtained by PCR amplification of the previous construct pCycT300-EGFP with primers: FW $5^{\prime}$-gc aag ctt cg gag gga gag agg aag aac- $3^{\prime}, \mathrm{RV} 3^{\prime}$-gc gtc gac tta ctt gta cag ctc gtc-5' and cloning as a HindIII/Sall fragment into pBFP$\mathrm{C} 1$ (Clontech). To control for correct folding of the fusion proteins we performed transcriptional co-activation assays of the HIV LTR using Tat-EBFP and the various cyclin constructs as described in [27].

The HL3T1 cell line used in this study were cultured in Dulbecco's modified Eagle's medium (DMEM) supplemented with $10 \%$ foetal bovine serum.

\subsubsection{Fluorescence resonance energy transfer (FRET).}

The presence of FRET indicates actual protein-protein interaction at distances in the range of the FRET length scale, the Förster radius $(R 0)$, defined as the distances at which FRET efficiency is $50 \%$. FRET is exploited in vivo by the use of the green fluorescent protein family of fluorophores. For the green fluorescent protein (GFP):blue fluorescent protein (BFP) pair $R_{0} \approx 4 \mathrm{~nm}$, this implies that simple colocalization of two proteins is not sufficient to yield energy transfer. Epithelial cells (HL3T1) were transiently transfected by the calcium phosphate method with the various expression plasmids fused to the optically-matched fluorescent tags in four-chambers of glass slides. Cells were fixed in $4 \%$ paraformaldehyde after $48 \mathrm{~h}$ and mounted directly in $70 \%$ glycerol for FRET analysis. FRET measurements were carried out as previously described by an epi-fluorescence Axioskop 2 Zeiss microscope mounting a 103 W HBO lamp, a $100 \times 1.3$ N.A. oil-immersion PlanNeofluar objective, and Nomarski optics [26,27]. FRET analysis was performed in two steps. Firstly, enhanced green fluorescent protein (EGFP) emission was collected by integrating the fluorescence signal around $520 \mathrm{~nm}$ (bandwidth $40 \mathrm{~nm}$ ) under EGFP excitation at $480 \mathrm{~nm}$ (wavelength selection was obtained by $40 \mathrm{~nm}$ band-pass filters, excitation power was $5 \mathrm{~W} / \mathrm{cm}^{2}$ ). Secondly, EGFP emission in the same frequency range was measured 


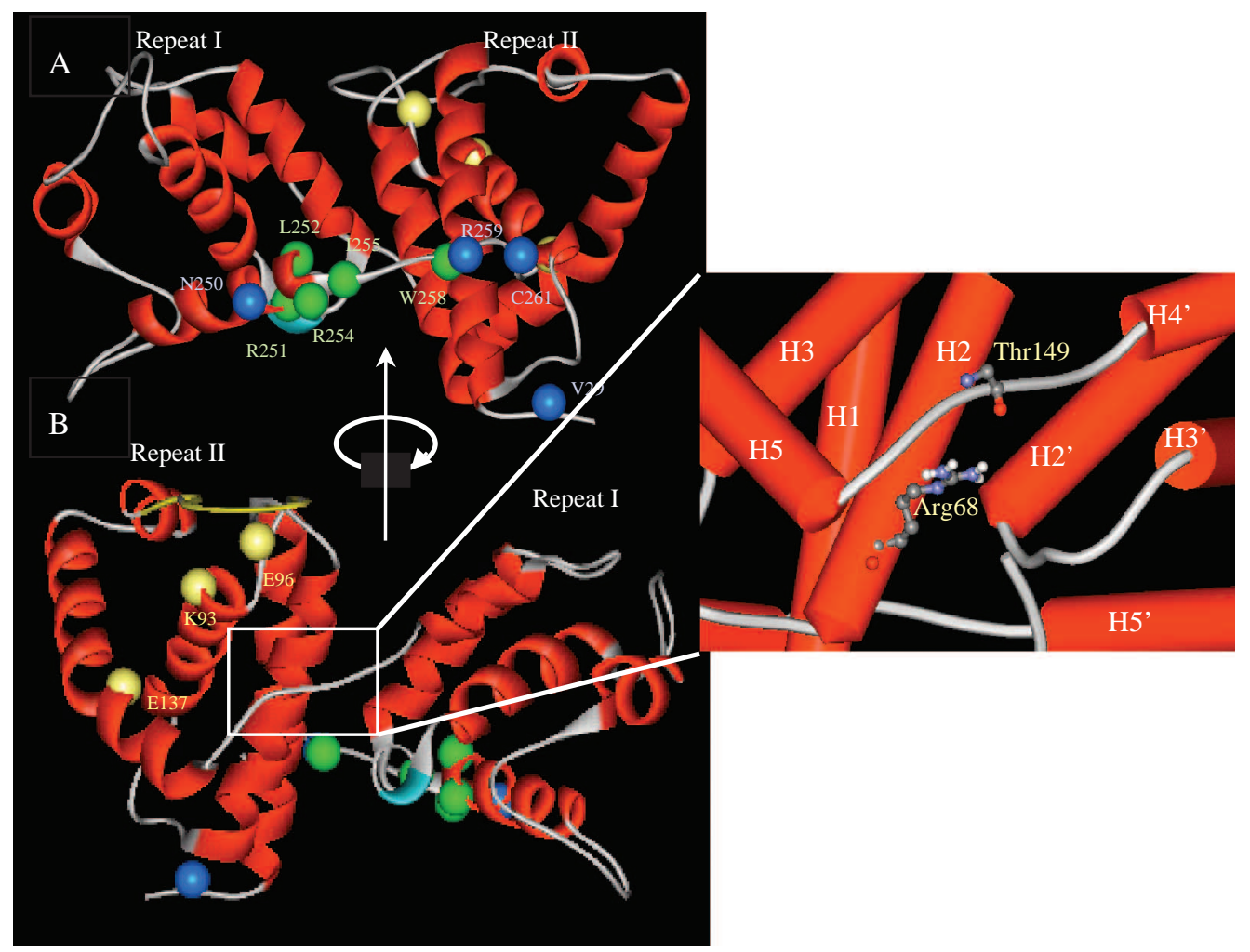

Figure 2. Modelling of CycT. (A) 3D structure of CycT1 as obtained from comparative modelling with CycH. C $\alpha$ of Val29, Asp250, Arg259 and Cys261, which are known to interact directly or indirectly with Tat are shown in light blue with ball representation. C $\alpha$ of Arg251, Leu252, Arg254, Ile255 and Trp258 are shown as green balls. (B) CDK9 binding region (the molecule is rotated $180^{\circ}$ with respect to A). C $\alpha$ of Lys93, Glu96 and Glu 137, involved in CDK9 recognition are represented in balls and sticks. Glu147 and the loop (marked in yellow) between $\mathrm{H} 3$ and $\mathrm{H} 4$ that may putatively participate in CDK9 binding are also shown. Inset: a close up showing the conserved interaction between Arg68 and the carbonyl moiety of the Thr149 backbone.

after excitation at $350 \mathrm{~nm}$ (power density $2 \mathrm{~W} / \mathrm{cm}^{2}$ and bandwidth $60 \mathrm{~nm}$ ). Background was detected out of the cell under study for each frame and subtracted from the relevant fluorescent signal. Following this procedure, the ratio between the two measured EGFP emissions (data taken following excitation at $350 \mathrm{~nm}$ divided by those at $480 \mathrm{~nm}$ ) provides the FRET signal. Fluorescence was collected by a PentaMax 512-EFT intensified CCD camera with detection times of the order of $0.1 \mathrm{~s}$ (in particular, for data taken under excitation at $350 \mathrm{~nm}$ they were five times longer than for those relative to $480 \mathrm{~nm}$ excitation). Data acquisition and analysis were performed with the Metamorph software from Universal Imaging Corporation, a subsidiary of Molecular Devices. When evaluating FRET ratios, emission intensities were scaled to take into account the different detection times.

\section{Results and discussion}

\subsection{Structural model}

3.1.1 Sequence alignment. Our alignment with the five templates spanning the respective cyclin box domains is shown in figure 1. We considered a 236 residues long fragment of CycT1 spanning from Phe27 to Ala263 that covers the protein portion involved in HIV-1 Tat and CDK9 binding. As can be observed from figure 1, all the gaps lie within loopy regions, and better conservation is obtained within the first domain.

As found for other members of the Cyclin family of proteins [4], the percent of identity between CycT1 and the templates is low (table 1). However, the characteristic cyclin box fold is structurally well preserved even at low degrees of sequence conservation. The high hydrophobic character of helix $\mathrm{H} 3$ that is surrounded by the other four helices in each repeat is clearly seen from figure 1 . The existence of gaps before and after helices $\mathrm{H} 4$ and $\mathrm{H}^{\prime}$, suggest either, the presence in CycT1 of longer loops or helical elements than in all the other cyclins. Site directed mutagenesis experiments on positions Lys93 and Glu96 [28,29], immediately before

Table 1. Percent of sequence identity between CycT1 and the templates obtained from the sequence alignment (figure 1) and root mean square deviation (RMSD) calculated per cyclin repeat and on the whole cyclin boxes. The end to end distance reported correspond to the distance between the first and last residue aligned for each protein.

\begin{tabular}{|c|c|c|c|c|c|}
\hline & $\mathrm{CycH}$ & CycA & TFIIB & v-Cyc & K-Cyc \\
\hline$\%$ Identity & 14 & 19 & 14 & 9 & 11 \\
\hline RMSD [Å] (Global) & 0.8 & 1.6 & 7.7 & 2 & 1.5 \\
\hline RMSD [A] Repeat I & 0.5 & 1.4 & 1.4 & 1.3 & 1.4 \\
\hline RMSD $[\AA]$ Repeat II & 0.9 & 1.7 & 1.5 & 1.4 & 1.6 \\
\hline $\begin{array}{l}\text { End to end distance } \\
{[\mathrm{nm}]}\end{array}$ & 1.4 & 2.6 & 5.6 & 2 & 2.3 \\
\hline
\end{tabular}


the first gap, demonstrates that $\mathrm{CDK}^{\prime}$ s binding site is located in this zone, between helix $\mathrm{H} 3$ and $\mathrm{H} 4$.

The $\mathrm{C}$ terminus motifs of $\mathrm{CycH}$ and $\mathrm{CycT} 1$ align without gaps after $\mathrm{H}^{\prime}$, at the end of the cyclin box. This segment contains the TRM (residues 250-262) of CycT1. Among all the templates, $\mathrm{CycH}$ presents the highest homology with CycT1 (figure 1).

3.1.2 3D-model. Based on the alignment of figure 1, a structural model of the protein was built (figure 2) using the structure of $\mathrm{CycH}$ as template. The $\mathrm{N}$ and $\mathrm{C}$ termini of the cyclin box lie on the first repeat, separated $11 \AA$ one from the other. The relative orientation of both repeats is stabilized by the highly conserved residue Arg68 [3], which is in good position to form H-bonds with Tyr49 backbone carbonyl moieties (figure 2). This interaction is conserved in all the templates, with the exception of TFIIB, and was reported to be crucial to fix helix $\mathrm{H} 2$ to the linker, and hence, the relative orientation between repeats I and II $[20,30]$. In fact, the cyclin box of TIIFB presents a glutamine instead of an arginine in the corresponding position. This change provokes the weakness of this strongly stabilizing interaction with the result that the relative orientation of both repeats is different from the rest of the cyclins.

Structural superposition performed on the whole molecules, or considering only one repeat at the time, indicate that the structural dispersion between all the cyclins considered and our model of CycT1 is indeed small (table 1) suggesting that any of the cyclin structures could be used as structural template obtaining practically identical results.

3.1.3 Consistency with experimental data. We then investigated the location of the residues of CycT1, which bind to its molecular partners (Tat, TAR and CDK9) and for which mutagenesis data are available in the literature. All the residues reported to participate in CDK9 binding to CycT1 (Lys93, Glu96 and Glu137) [29,30] were solventexposed and in close proximity (figure 2). These residues are also highly conserved along the cyclin family [3] (figure 1). Lys93 and Glu96 are located at the end of helix $\mathrm{H} 3$, whilst Glu137 is located in the middle of H5. We further notice that other charged residues such as Lys99/100 Glu102 located in the loop connecting H3 with $\mathrm{H} 4$ and Glu147 within H5 are also exposed to the solvent and near to the putative CDK9 binding region, so they might also play a role in CDK9 binding.

All the residues known to bind to HIV-1 Tat or TAR [8] (figure 2(A)) resulted exposed to the solvent. Asn250, Arg251 and Leu252 are located in last turn of helix H5', while Arg254, Ile245, Trp258, Arg259 and Cys261 lie in coil conformation.

This conformation is strongly supported by recently reported proteolysis experiments [31] which showed that (i) CycT1 TRM is susceptible to digestion and therefore, is in a flexible conformation. (ii) CycT1 is cleaved after Leu252, indicating that this is the last residue in ordered (helical) conformation, which is in nice agreement with our theoretical predictions.

Finally, Val29, whose mutation to Leu was reported to render human CycT1 active for equine Tat transactivation in EIAV [32], is also exposed and oriented towards the TRM.

\subsection{Validation of the model}

As stated above, the cyclin box fold is highly conserved even at low identity levels. Therefore, the largest assumption in our model is that in CycT1 the relative orientation of both the cyclin repeats that determine the distance between $\mathrm{N}$ and $\mathrm{C}$ termini is very similar to that of $\mathrm{CycH}$. To validate this proposal, we performed in vivo FRET experiments. FRET has been already successfully used in the last few years to detect CycT1-protein interaction within living cells, using the GFP family of fluorophores [15,26]. Here we used this technique to estimate the proximity between the $\mathrm{N}$ and $\mathrm{C}$ termini of the protein, which may sensibly vary with the relative orientation of the cyclin repeats.

As a first attempt, we tried to provide an estimation of this proximity by intra-molecular FRET in cells cotransfected with plasmids expressing CycT1 tagged at the $\mathrm{N}$-terminus with the BFP and at the C-terminus with EGFP. FRET image analysis of individually transfected cells are shown in figure 3(A), column 3. FRET analysis was performed by comparing EGFP emission at $520 \mathrm{~nm}$ (the peak wavelength of EGFP emission), following BFP excitation at $350 \mathrm{~nm}$ (figure 3(A), panels in row c), with that following excitation at $480 \mathrm{~nm}$ of the same cells (figure 3(A), panels in row b). As it can be seen from figure 3 , in this case no FRET signal was obtained.

We then proceed to investigate this proximity by intermolecular FRET, taking advantage of the fact the CycT1's TRM is located around position 261, within the C-terminal helix after the cyclin box. We therefore co-transfected human HL3T1 cells with plasmids expressing Cyclin T1, tagged at the N-termini with EGFP, and Tat tagged at the C-termini with BFP. Most cells transfected with EGFPCyclin T1:Tat-BFP (figure 3(A), panel b1) showed the characteristic nuclear punctuated pattern of Cyclin T1 [26,33]. Samples expressing both EGFP-CycT1 and TatBFP scored positive for FRET. This suggests that the distance between the N-terminus of CycT1 TRM and the Cterminus of CytT1 is in the range of that of the template (table 1).

Based on these findings, we tested whether fusing GFP at the $\mathrm{C}$ terminus of CytT1 provides FRET with Tat-BFP. Cells transfected with CycT300-EGFP:Tat-BFP (figure 3, column 2) showed a nucleo-cytoplasmic distribution of CycT300-EGFP at $520 \mathrm{~nm}$ under excitation at $480 \mathrm{~nm}$. The difference of distribution of the protein with respect to the wild-type CycT1 reflected the lack of the carboxyterminal domain of the protein, which was responsible for the punctuated nuclear localization through binding to the PML protein [15]. EGFP emission at $520 \mathrm{~nm}$, following BFP excitation at $350 \mathrm{~nm}$, scored positive for FRET, 
A
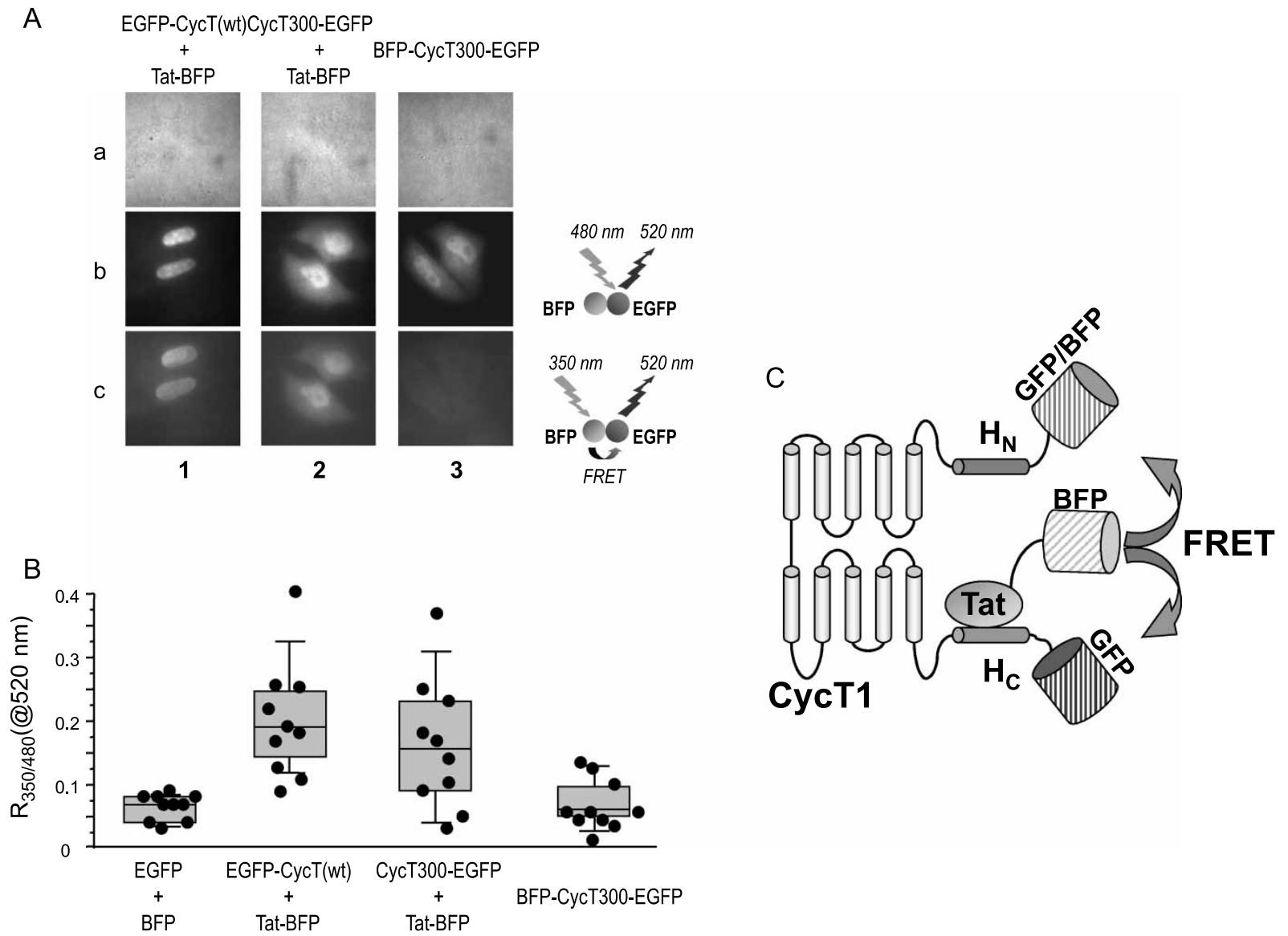

Figure 3. FRET analysis in vivo. (A) FRET visualization in human cells. The plasmid constructs indicated on top of each column were transfected in HL3T1 cells (a HeLa derivative cell line carrying an integrated LTR CAT cassette). Transfected cells were visualized by transmitted light in Normarski configuration (panels in row a), by excitation at $480 \mathrm{~nm}$ and collection at $520 \mathrm{~nm}$, showing direct EGFP excitation (panels in row b), and by excitation at $350 \mathrm{~nm}$ and collection at $520 \mathrm{~nm}$, showing EGFP fluorescence after BFP excitation, indicating FRET (panels in row c). (B) FRET quantification. Fluorescent emission at $520 \mathrm{~nm}$ from individual cells transfected with the indicated constructs was recorded after excitation at 480 or $350 \mathrm{~nm}$, and integrated intensities over the whole cells were evaluated. Plotted values (indicated by dots) represent the ratio between these two measurements: higher values indicate more efficient FRET between BFP and EGFP. Ten consecutively analysed cells were considered for each transfection; both their individual fluorescence ratios and their percentile box plot distribution are shown. Horizontal lines from top to bottom mark the 10-, 25-, 50-, 75-, 90-th percentiles, respectively. (C) Schematic representation of the putative structure of the N-terminus of Cyclin T1 with respect to the binding of Tat.

substantiating the fact that Tat-BFP was within $4 \mathrm{~nm}$ to the $\mathrm{N}$ and $\mathrm{C}$ termini of $\mathrm{CycT}_{(1-300)}$.

The detailed, quantitative analysis of at least 10 cells expressing for the protein pairs is presented in figure 3B, showing the percentile distribution of FRET values. We consider the signal from cells transfected with EGFP and BFP (background) as baseline.

\section{Concluding remarks}

The recruitment of P-TEFb by Tat through the specific binding of CycT1 is a crucial step in the replication cycle of HIV-1. However, although a considerable amount of biological data is available, a detailed structural characterization, which may help to rationalize these data and constitute the basis for future experiments, is still lacking.

We present here a theoretical structure of the $\mathrm{N}$ terminus of CycT1 based on the structure of its human transcriptional homologue $\mathrm{CycH}$ and of other cyclin proteins with known structure. Due to the low level of sequence identity between target and template (figure 1(B)), the results of the homology modelling are confronted with site directed mutagenesis data present in literature. The residues involved in the CycT1: Tat interaction (from aa 250-261) turned out to be located at the $\mathrm{H} 5$ 'term-coil-Hc term segment and solvent-exposed (figure 2), in good agreement with proteolysis data [31]. Although we were confident with the gross structural determinants of the cyclin box region of the model, also considering that this fold is highly conserved, we wish to point out that the orientation of the $\mathrm{N}$ and $\mathrm{C}$ termini remained rather uncertain, depending on the choice of the template (table 1). Thus, FRET experiments were carried out in this work to probe the spatial arrangement of the $\mathrm{N}$ and $\mathrm{C}$ terminal ends of the protein. By measuring FRET between EGFP-CycT1/Tat-BFP and CycT1-EGFP/Tat-BFP complexes we are able to confirm spatial proximity of these two regions in living cells (figure 3 ).

In conclusion, the model presented here is consistent with all experimental data up to the best of our knowledge. However, it must be kept in mind that it suffers the obvious limitations and uncertainties of comparative modelling, 
particularly at low sequence identity levels. Still, we believe that it may be useful to test working ideas and inspire new biochemical experiments aimed to clarify structural features of protein-protein interactions in which CycT1 is involved.

\section{Acknowledgements}

This work was supported by grants from the National Research Programme on AIDS of the Istituto Superiore di Sanità to M.G. and A.M., from the Ministero Istruzione Universita' e Ricerca to M.G. and A.M., from the Human Frontier Science Program to A.M., from COFIN-MURST and Regione Friuli Venezia Giulia to S.P. and P.C. and from Fondazione Cariparo to S.P.

\section{References}

[1] Lew, D.J. and Kornbluth, S., 1996, Regulatory roles of cyclin dependent kinase phosphorylation in cell cycle control. Curr. Opin. Cell. Biol., 8, 795-804.

[2] Dynlacht, B.D., 1997, Regulation of transcription by proteins that control the cell cycle. Nature, 389, 149-152.

[3] Bazan, J.F., 1996, Helical fold prediction for the cyclin box. Proteins, 24, 1-17.

[4] Noble, M.E., Endicott, J.A., Brown, N.R. and Johnson, L.N., 1997, The cyclin box fold: Protein recognition in cell-cycle and transcription control. Trends Biochem. Sci., 22, 482-487.

[5] Wei, P., Garber, M.E., Fang, S.M., Fischer, W.H. and Jones, K.A., 1998, A novel CDK9-associated C-type cyclin interacts directly with HIV-1 Tat and mediates its high-affinity, loop-specific binding to TAR RNA. Cell, 92, 451-462.

[6] Bieniasz, P.D., Grdina, T.A., Bogerd, H.P. and Cullen, B.R., 1998, Recruitment of a protein complex containing Tat and cyclin $\mathrm{T} 1$ to TAR governs the species specificity of HIV-1 Tat. EMBO J., 17, $7056-7065$

[7] Fujinaga, K., Cujec, T.P., Peng, J., Garriga, J., Price, D.H., Grana, X. and Peterlin, B.M., 1998, The ability of positive transcription elongation factor $\mathrm{B}$ to transactivate human immunodeficiency virus transcription depends on a functional kinase domain, cyclin T1, and Tat. J. Virol., 72, 7154-7159.

[8] Garber, M.E., Wei, P., KewalRamani, V.N., Mayall, T.P., Herrmann, C.H., Rice, A.P., Littman, D.R. and Jones, K.A., 1998, The interaction between HIV-1 Tat and human cyclin T1 requires zinc and a critical cysteine residue that is not conserved in the murine CycT1 protein. Genes Dev., 12, 3512-3527.

[9] Zhou, Q., Chen, D., Pierstorff, E. and Luo, K., 1998, Transcription elongation factor P-TEFb mediates Tat activation of HIV-1 transcription at multiple stages. EMBO J., 17, 3681-3691.

[10] Kanazawa, S., Okamoto, T. and Peterlin, B.M., 2000, Tat competes with CIITA for the binding to P-TEFb and blocks the expression of MHC class II genes in HIV infection. Immunity, 12, 61-70.

[11] Barboric, M., Nissen, R.M., Kanazawa, S., Jabrane-Ferrat, N. and Peterlin, B.M., 2001, NF-kappaB binds P-TEFb to stimulate transcriptional elongation by RNA polymerase II. Mol. Cell., 8, 327-337.

[12] Eberhardy, S.R. and Farnham, P.J., 2002, Myc recruits P-TEFb to mediate the final step in the transcriptional activation of the cad promoter. J. Biol. Chem., 277, 40156-40162.

[13] Fong, Y.W. and Zhou, Q., 2000, Relief of two built-in autoinhibitory mechanisms in $\mathrm{P}-\mathrm{TEFb}$ is required for assembly of a multicomponent transcription elongation complex at the human immunodeficiency virus type 1 promoter. Mol. Cell. Biol., 20, 5897-5907.

[14] Taube, R., Lin, X., Irwin, D., Fujinaga, K. and Peterlin, B.M., 2002, Interaction between $\mathrm{P}-\mathrm{TEFb}$ and the $\mathrm{C}$-terminal domain of RNA polymerase II activates transcriptional elongation from sites upstream or downstream of target genes. Mol. Cell. Biol., 22, $321-331$.

[15] Marcello, A., Ferrari, A., Pellegrini, V., Pegoraro, G., Lusic, M., Beltram, F. and Giacca, M., 2003, Recruitment of human cyclin T1 to nuclear bodies through direct interaction with the PML protein. EMBO J., 22, 2156-2166.

[16] Schulze-Gahmen, U., Jung, J.U. and Kim, S.H., 1999, Crystal structure of a viral cyclin, a positive regulator of cyclin-dependent kinase 6. Struct. Fold Des., 7, 245-254.

[17] Jeffrey, P.D., Tong, L. and Pavletich, N.P., 2000, Structural basis of inhibition of CDK-cyclin complexes by INK4 inhibitors. Genes Dev., 14, 3115-3125.

[18] Russo, A.A., Jeffrey, P.D., Patten, A.K., Massague, J. and Pavletich, N.P., 1996, Crystal structure of the p27Kip1 cyclin-dependentkinase inhibitor bound to the cyclin A-Cdk2 complex. Nature, 382, $325-331$

[19] Nikolov, D.B., Chen, H., Halay, E.D., Usheva, A.A., Hisatake, K., Lee, D.K., Roeder, R.G. and Burley, S.K., 1995, Crystal structure of a TFIIB-TBP-TATA-element ternary complex. Nature, 377, $119-128$

[20] Andersen, G., Poterszman, A., Egly, J.M., Moras, D. and Thierry, J.C., 1996, The crystal structure of human cyclin H. FEBS Lett., 397, 65-69.

[21] Notredame, C., Higgins, D.G. and Heringa, J., 2000, T-Coffee: A novel method for fast and accurate multiple sequence alignment. J. Mol. Biol., 302, 205-217.

[22] Parada, C.A. and Roeder, R.G., 1996, Enhanced processivity of RNA polymerase II triggered by Tat-induced phosphorylation of its carboxy-terminal domain. Nature, 384, 375-378.

[23] Ivanov, D., Kwak, Y.T., Nee, E., Guo, J., Garcia-Martinez, L.F. and Gaynor, R.B., 1999, Cyclin T1 domains involved in complex formation with Tat and TAR RNA are critical for tat-activation. J. Mol. Biol., 288, 41-56.

[24] Sali, A. and Blundell, T.L., 1993, Comparative protein modelling by satisfaction of spatial restraints. J. Mol. Biol., 234, 779-815.

[25] Fiser, A., Do, R.K. and Sali, A., 2000, Modeling of loops in protein structures. Protein Sci., 9, 1753-1773.

[26] Marcello, A., Cinelli, R.A., Ferrari, A., Signorelli, A., Tyagi, M., Pellegrini, V., Beltram, F. and Giacca, M., 2001, Visualization of in vivo direct interaction between HIV-1 TAT and human cyclin T1 in specific subcellular compartments by fluorescence resonance energy transfer. J. Biol. Chem., 276, 39220-39225.

[27] Marcello, A., Ferrari, A., Pellegrini, V., Pegoraro, G., Lusic, M., Beltram, F. and Giacca, M., 2003, Recruitment of human cyclin T1 to nuclear bodies through direct interaction with the PML protein EMBO J., 22, 2156-2166.

[28] Fraldi, A., Licciardo, P., Majello, B., Giordano, A. and Lania, L., 2001, Distinct regions of cyclinT1 are required for binding to CDK9 and for recruitment to the HIV-1 Tat/TAR complex. J. Cell. Biochem., 81, 247-253.

[29] Fujinaga, K., Irwin, D., Geyer, M. and Peterlin, B.M., 2002, Optimized chimeras between kinase-inactive mutant Cdk9 and truncated cyclin T1 proteins efficiently inhibit Tat transactivation and human immunodeficiency virus gene expression. J. Virol., 76 10873-10881.

[30] Andersen, G., Busso, D., Poterszman, A., Hwang, J.R., Wurtz, J.M., Ripp, R., Thierry, J.C., Egly, J.M. and Moras, D., 1997, The structure of cyclin $\mathrm{H}$ : common mode of kinase activation and specific features. EMBO J., 16, 958-967.

[31] Das, C., Edgcomb, S.P., Peteranderl, R., Chen, L. and Frankel, A.D., 2004, Evidence for conformational flexibility in the Tat-TAR recognition motif of cyclin T1. Virology, 318, 306-317.

[32] Taube, R., Fujinaga, K., Irwin, D., Wimmer, J., Geyer, M. and Peterlin, B.M., 2000, Interactions between equine cyclin T1, Tat, and TAR are disrupted by a leucine-to-valine substitution found in human cyclin T1. J. Virol., 74, 892-898.

[33] Herrmann, C.H. and Mancini, M.A., 2001, The Cdk9 and cyclin T subunits of TAK/P-TEFb localize to splicing factor-rich nuclear speckle regions. J. Cell. Sci., 114, 1491-1503.

[34] Price, D.H., 2000, P-TEFb, a cyclin-dependent kinase controlling elongation by RNA polymerase II. Mol. Cell. Biol., 20, 2629-2634. 


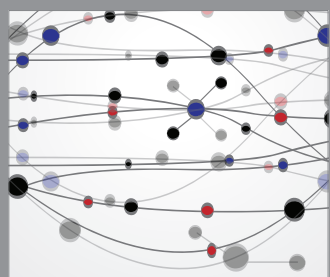

The Scientific World Journal
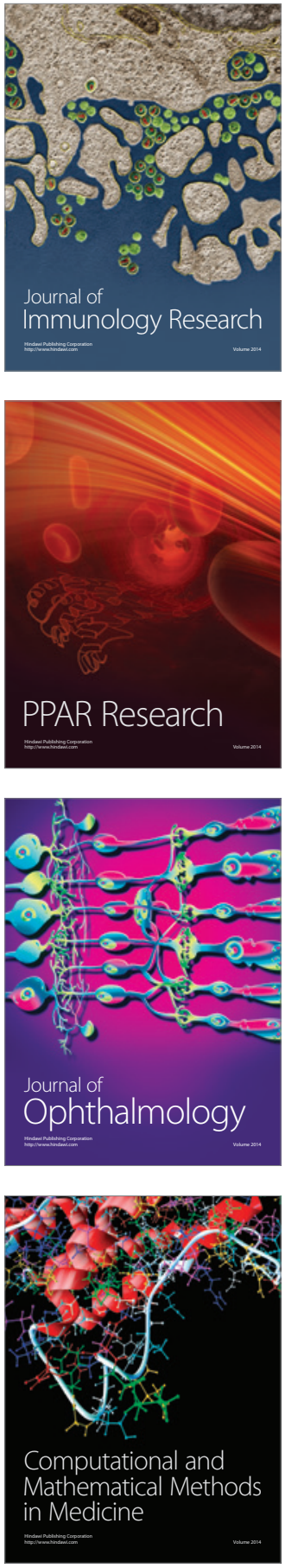

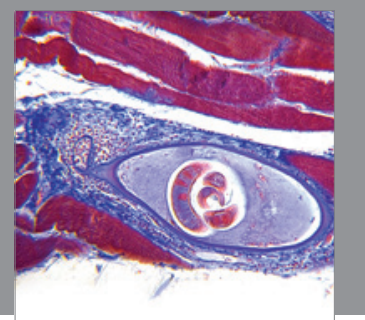

Gastroenterology

Research and Practice
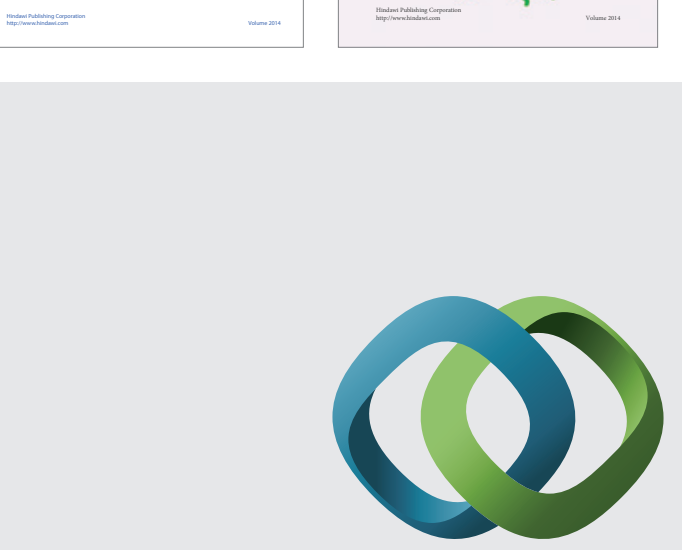

\section{Hindawi}

Submit your manuscripts at

http://www.hindawi.com
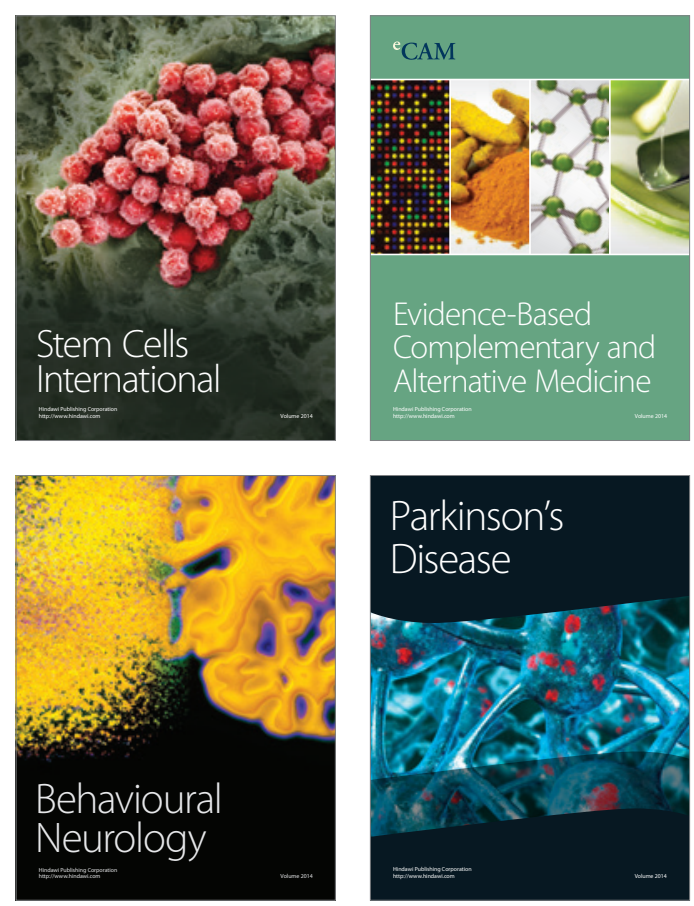

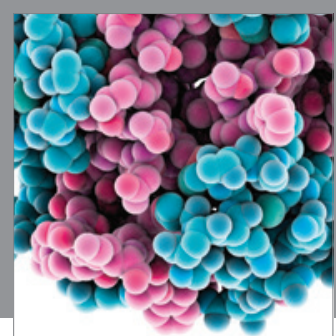

Journal of
Diabetes Research

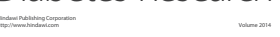

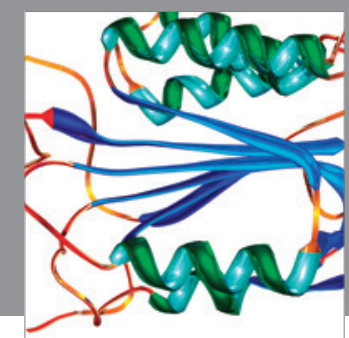

Disease Markers
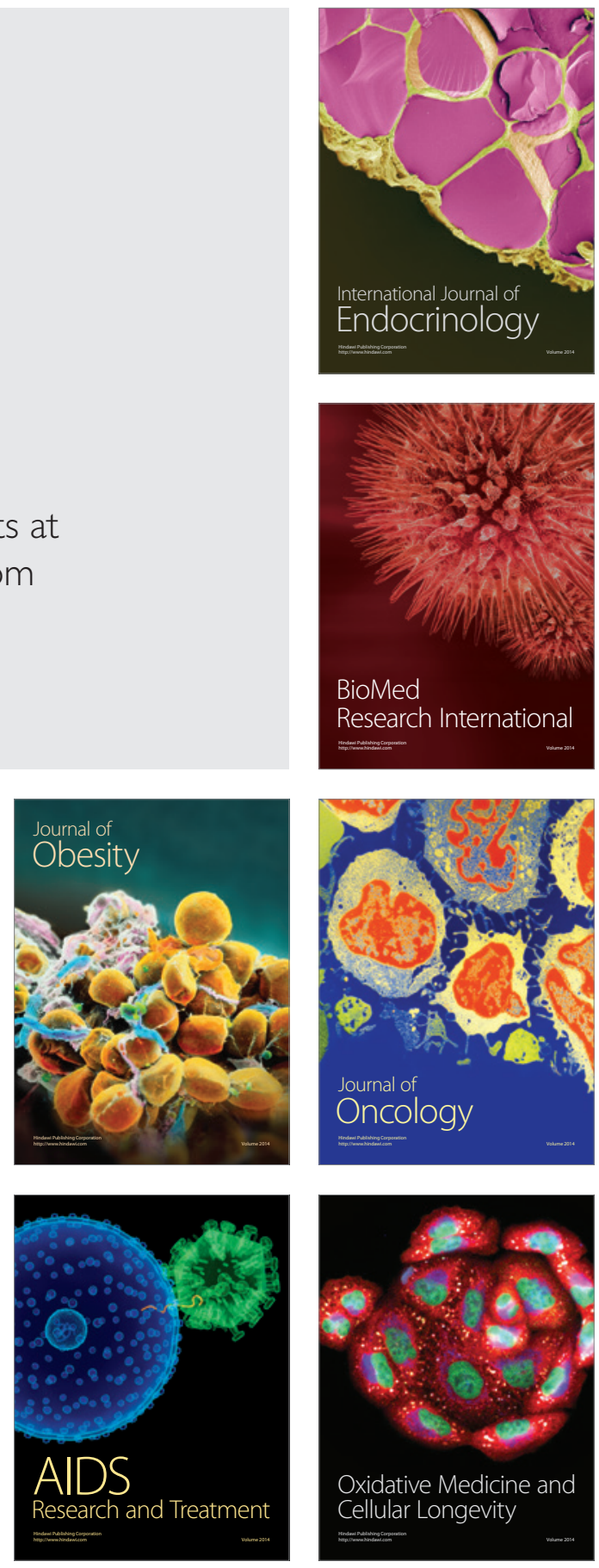